\title{
Period Doubling Cascade in Diffusion Flames
}

\author{
Milan Miklavčič ${ }^{*}$ \\ Department of Mathematics, Michigan State University, East Lansing, MI 48824, USA \\ to appear in 2006 Combustion Theory Modelling
}

\begin{abstract}
Here it is shown that chaotic oscillations can appear after a series of period doublings in radiating diffusion flames when the activation temperature is high enough. It is also shown that period doubling cascades appear typically in very small regions and that they may not be observable if one starts with small perturbations of a steady flame.
\end{abstract}

Keywords: Diffusion flames, Hopf bifurcation, stable oscillations, stable limit cycle, chaos, period doubling

\section{Introduction}

Oscillations in diffusion flames have been studied for a long time. Early studies by Matalon, Kim and coworkers used high activation energy asymptotic techniques to study the corresponding linear stability problem, see $[2,9,10,11,14]$. Vance et al. [17] studied numerically the eigenvalues of the complete linearized system. In numerous numerical experiments it was found [13] that linearly growing oscillations in diffusion flames lead to flame extinction if radiation is not accounted for.

Sohn et al. [15] were the first to show that, at one special set of parameters, linearly growing oscillations can develop into a stable oscillatory flame when the nonlinear optically thin radiation approximation $R D\left(T^{4}-T_{0}^{4}\right)$ is used. A numerical investigation by Miklavčič et al. [13] of oscillations in diffusion flames under a wide range of parameters shows when one can expect the appearance of simple stable periodic oscillations (SPO), see Figure 1. In particular, they [13] showed that radiation causes the appearance of linearly unstable states at large Damköhler numbers on the tail of the S-curve first. If the activation temperature and radiation are small enough, then perturbations of all those unstable states lead to SPO. Hence there can be no periodic doubling cascades in such cases.

If, on the other hand, the activation temperature is large enough, then SPO appear only near the Damköhler number where transition from linearly stable to linearly unstable states occurs i.e. near the point where the supercritical Hopf bifurcation occurs. Further away from this point, it was found [13] that perturbations of unstable states lead to flame extinction. Somebody working in dynamical systems would perhaps expect to find a period doubling cascade on the border between the two regions. However period doubling cascades

*e-mail: milan@math.msu.edu 


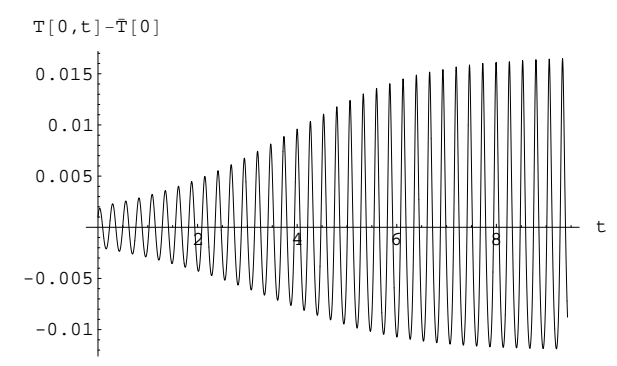

Figure 1: Evolution of typical simple stable periodic oscillations (SPO).

were never observed when the regions of SPO were determined in [13]. Christiansen et al. [3] were not even able to find the SPO part of the period doubling cascade in their recent numerical study. Here it is explained why. In short, the main reasons are (1) the region where the period doubling cascade occurs is relatively small and (2) small perturbations of the unstable steady solutions are usually not in the basin of attraction of stable periodic solutions with higher periods.

The main purpose of this paper is to show when and how one can one find period doubling cascades in diffusion flames. We will not focus too much on analyzing the cascade per se. Period doubling cascades have been studied in detail in all sort of settings $[5,8,12]$. For example, Feigenbaum speculated [4] that certain ratios of parameters where period doublings occur converge to a universal constant. Using the data presented here one can calculate the first two numbers of the infinite sequence. These two numbers are of the same order of magnitude as the Feigenbaum's universal constant, however, in this paper there was no attempt made to find the actual limit.

\section{Mathematical model}

It is assumed that fuel issues from the large reservoir behind a porous wall at $x=-1$
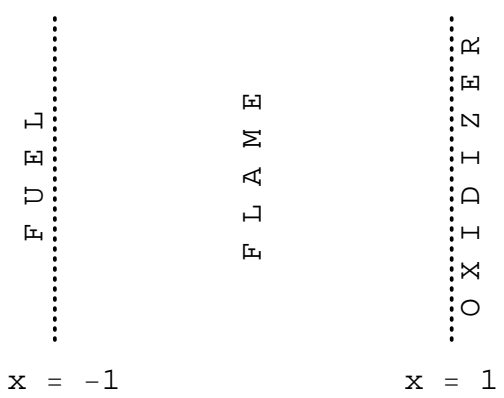

Figure 2: The one-dimensional diffusion flame between two porous walls.

and that the oxidizer diffuses from the free stream through the porous wall at $x=1$, see Figure 2. The equations governing the flame evolution over time $t>0$ can be written as

$$
\frac{\partial T}{\partial t}=\frac{\partial^{2} T}{\partial x^{2}}+w-R D\left(T^{4}-T_{0}^{4}\right)
$$




$$
\begin{aligned}
& L \frac{\partial Y_{o}}{\partial t}=\frac{\partial^{2} Y_{o}}{\partial x^{2}}-w, \\
& L \frac{\partial Y_{f}}{\partial t}=\frac{\partial^{2} Y_{f}}{\partial x^{2}}-w .
\end{aligned}
$$

Here $T=T(x, t)$ denotes the temperature, $Y_{o}$ is the oxidizer mass fraction and $Y_{f}$ gives the fuel mass fraction of the mixture. $L$ is the Lewis number, taken to be the same for the fuel and the oxidizer, and $R$ is the ratio of characteristic chemical and radiation time scales. In the limit as $R \rightarrow 0$, chemistry dominates and radiation becomes unimportant. The nondimensional reaction term $w$ for the one-step reaction is given by

$$
w=D Y_{o} Y_{f} e^{-T_{a} / T}
$$

where $D$ is the Damköhler number and $T_{a}$ is the activation temperature. The boundary conditions at the porous walls are:

$$
\begin{array}{ll}
\text { at } x=-1: & T=T_{0}, Y_{f}=1, Y_{o}=0, \\
\text { at } x=+1: & T=T_{0}, Y_{f}=0, Y_{o}=1 .
\end{array}
$$

This model has been studied extensively before [13]. In particular, nondimensionalization was carried out exactly as in Sohn et al. [15]. A slightly different configuration is preferred by Matalon and his coworkers [1, 2]. Christiansen et al. [3] added many complicated real-world influences. For a discussion of radiative loss see T'ien [16]. They all provide excellent physical descriptions of diffusion flames and cite many references.

The equations used by Sohn et al. [15] are equivalent to the above equations (1-6) when $T_{a}=5, T_{0}=0.1$ and $L=1$. Here it will be also assumed that $L=1, T_{0}=0.1$.

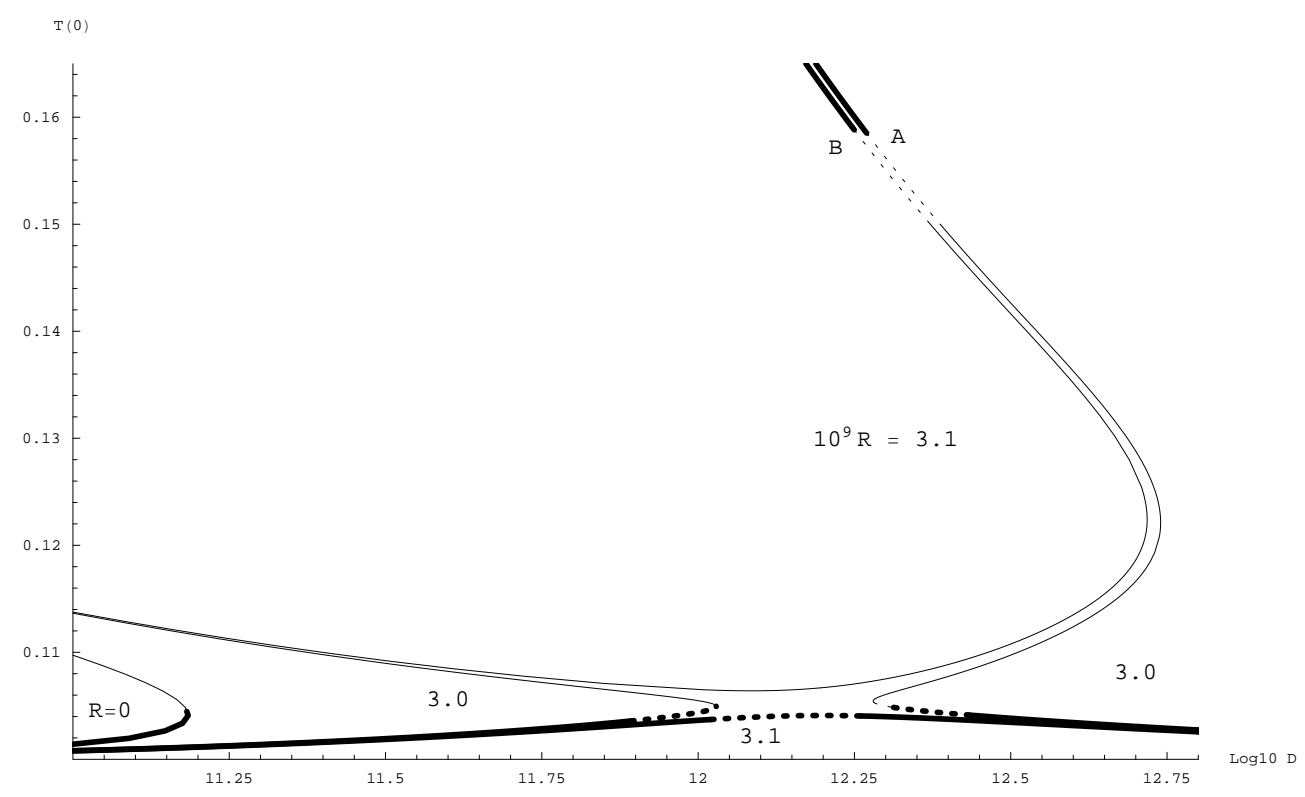

Figure 3: Creation of an island at $T_{a}=3$. At A and B supercritical Hopf bifurcations occur.

Analysis of the model starts with finding steady solutions of (1-6). Graphs of $T[0]$ of steady solutions vs $D$ give various curves. When $R=0$ one gets the classical S-curve, 
when $R$ is small the top of the S-curve gets pushed down, creating a tail [13]. As $R$ increases an island is created provided that $T_{a}>1$ [13]. For each steady solution one can find the leading eigenvalue of the linearized system, which determines the stability of the steady solution. Thick lines are used to denote stable solutions and thin lines to denote unstable solutions. When the leading eigenvalue is complex the line is dashed. When the real part of the complex eigenvalue changes sign a Hopf bifurcation usually occurs. When the Hopf bifurcation is supercritical, SPO evolve out of small perturbations of unstable steady solutions that are close to the bifurcation point. See Figure 3 for an example. The evolution of small perturbations of unstable steady solutions that are further away from the bifurcation point can follow various scenarios. In numerous calculations in [13] the outcome was always either SPO or flame extinction. Here it is shown that period doubling can occur too.

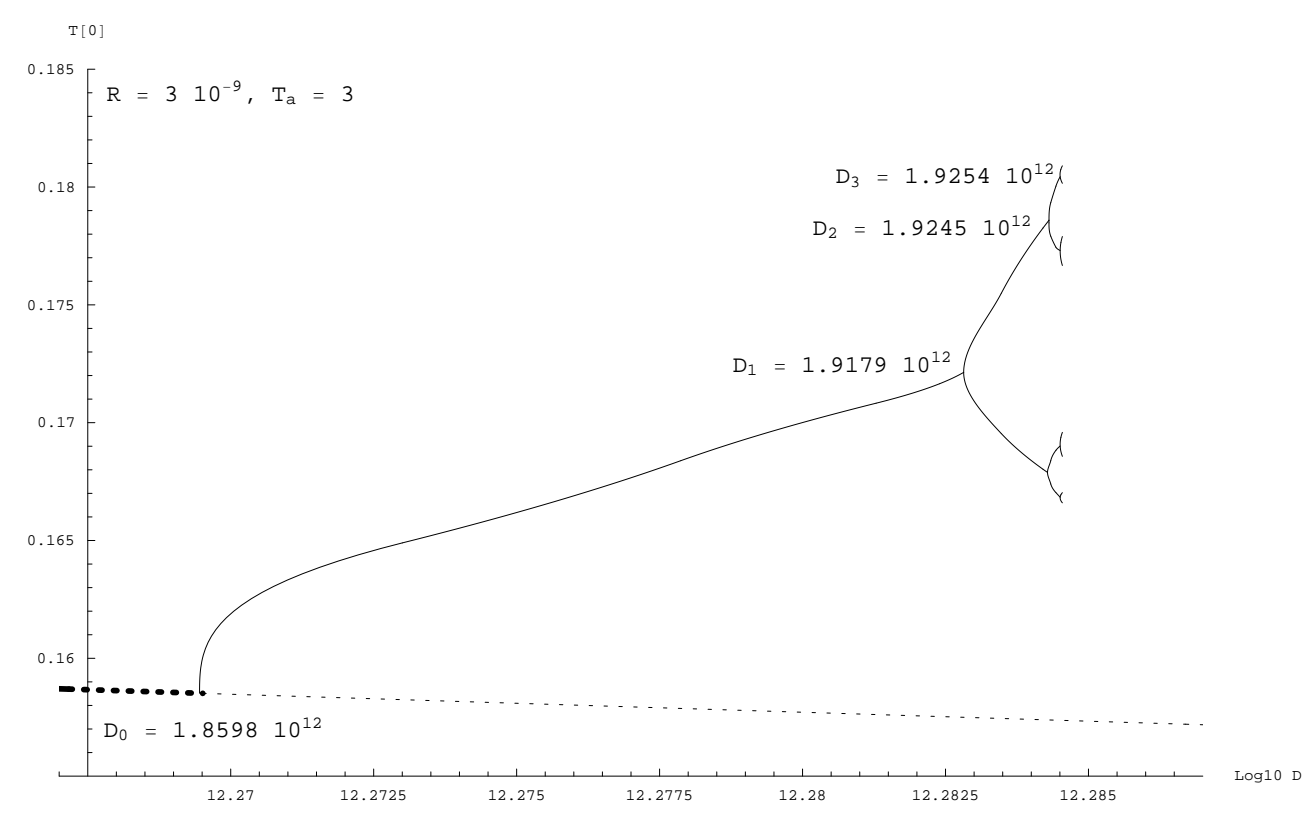

Figure 4: Period doubling cascade. The thin solid line shows here the peaks of temperature oscillations.

To be more specific consider Figure 3. In [13] it was reported that SPO occur near point $\mathrm{B}$ and that perturbations of unstable steady solutions a bit further away from $\mathrm{B}$ lead to flame extinction. When one zooms in on the region of transition nothing different happens. So, up to some $D^{*}$, perturbation of unstable solutions lead to SPO and, after $D^{*}$, they lead to flame extinction. At first glance the same happens at point A. However, when one zooms in on the region of transition, one finds a tiny region of oscillations with a doubled period in between. This is discussed in detail in the next section.

\section{Period doubling cascade}

Let us fix $R=3 \times 10^{-9}$ and $T_{a}=3$. A supercritical Hopf bifurcation occurs at $D_{0}=$ $1.8598 \times 10^{12}$ - at point A in Figure 3. Zooming in on the neighborhood of point A gives Figure 4 . The thick dashed line to the left of $D_{0}$ represents stable steady solutions. The 


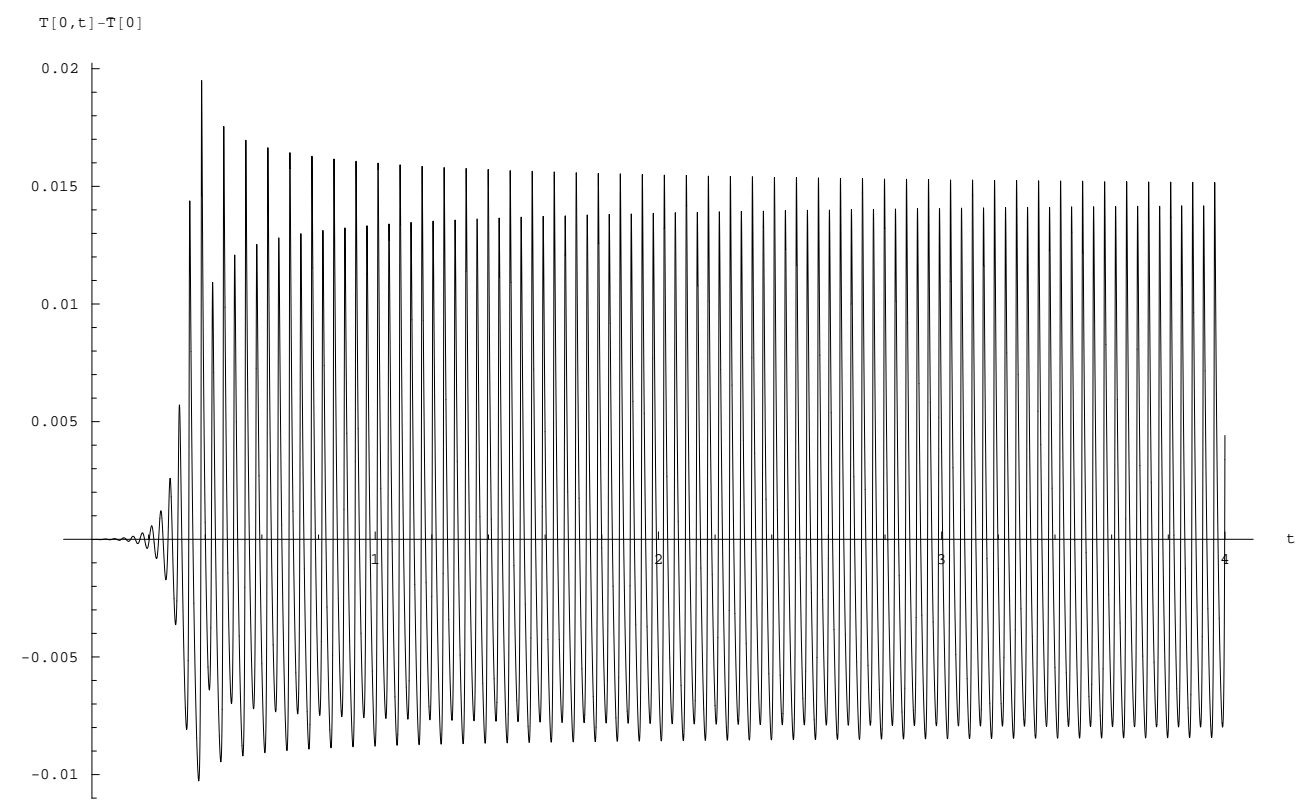

Figure 5: $D=1.9180 \times 10^{12}$

thin dashed line to the right of $D_{0}$ represents unstable steady solutions. In both cases the leading eigenvalue is complex. Between $D_{0}$ and $D_{1}$ small perturbations of steady solutions evolve into stable periodic solutions. The thin curve between $D_{0}$ and $D_{1}$ is the maximum temperature of stable periodic solutions. The period of oscillations is about 0.04 and slightly increases with $D$.

At $D_{1}=1.9179 \times 10^{12}$, every second peak of the stable periodic solution gets a bit larger and the one in between a bit smaller. See Figure 5. Hence the period doubles. The larger peak grows and the smaller peak gets smaller as $D$ increases. These two peaks create the two branches starting at $D_{1}$ in Figure 4.

Observe the overshooting in Figure 5. As $D$ increases, this overshooting increases rapidly. When $D>D^{*}=1.9208 \times 10^{12}$, the overshooting becomes too large - resulting in flame extinction. That is, when $D$ is bigger than $D^{*}$, small perturbations of steady solutions are no longer in the basins of attraction of stable periodic solutions. When $D_{0}<D<D^{*}$, periodic solutions were obtained by starting with a small perturbation of a steady solution. To obtain periodic solutions for $D>D^{*}$, the starting perturbation was chosen to be a periodic solution at some $D<D^{*}$ and then $D$ was increased past $D^{*}-$ oscillations stabilize at the new $D>D^{*}$ and flame extinction is avoided.

At $D_{2}=1.9245 \times 10^{12}$, every second larger peak gets larger and the one in between gets smaller. The smaller peaks separate similarly. See Figure 6. Thus the period doubles again. It is about 0.16 . The four branches starting at $D_{2}$ in Figure 4 show the sizes of the four peaks. Plotting $Y_{o}$ vs $T$ and omitting the initial transition phase gives Figure 7 .

At a slightly bigger $D_{3}=1.9254 \times 10^{12}$ the period doubles again - just like at $D_{1}$ and $D_{2}$.

At $D>1.9255 \times 10^{12}$, things start to change rapidly. At $D=1.9257 \times 10^{12}$, oscillations already seem quite chaotic. At $D=1.9260 \times 10^{12}$, some interesting behavior shows. In Figure 8 there is a "long" stretch of oscillations with period $2 \times 0.04$ in the middle which 


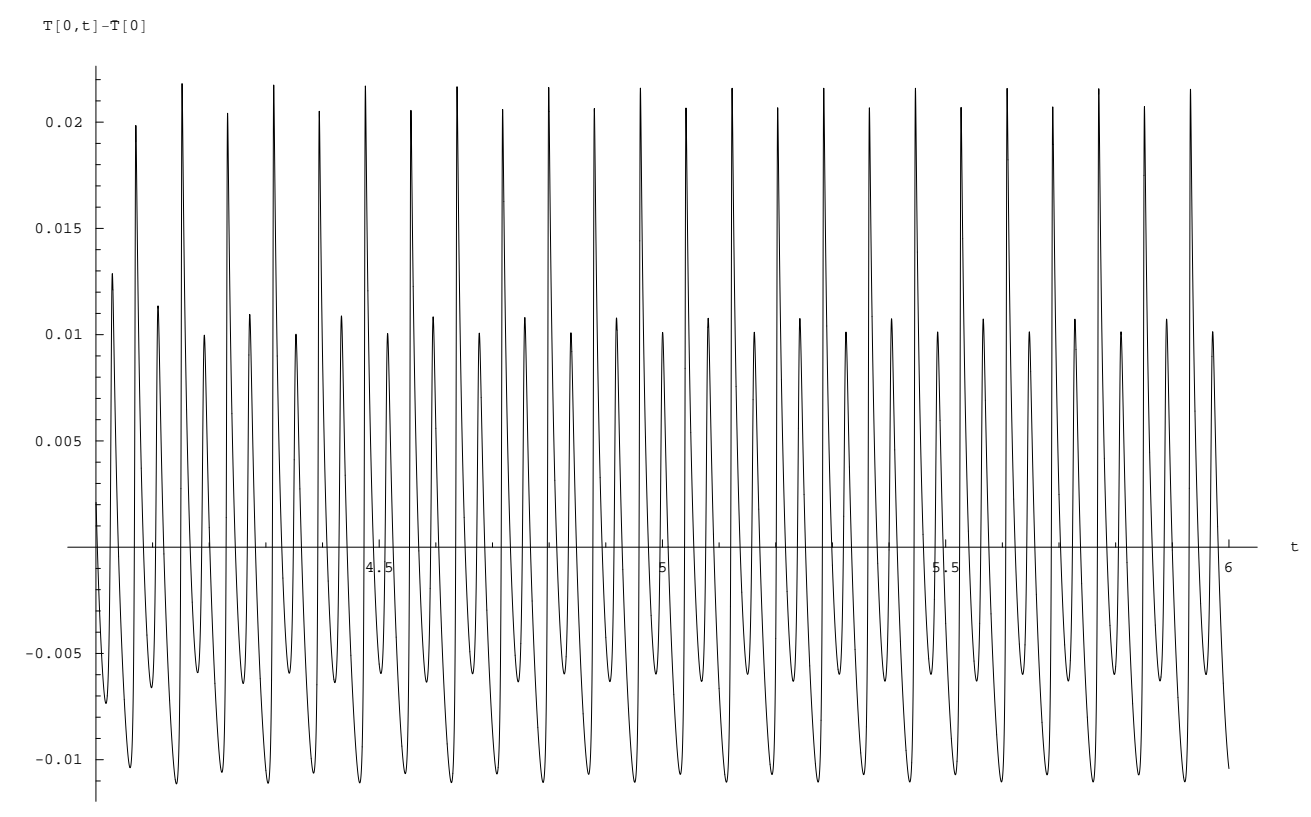

Figure $6: D=1.9245 \times 10^{12}$

is followed by several oscillations with period $6 \times 0.04$. If one looks at $Y_{o}$ vs $T$ for $t>4.5$ one gets Figure 9. There are 8 rings in Figure 9, let the smallest one be 1 . The middle part in Figure 8 corresponds to the repeating ring pattern 27 in Figure 9. The last part in Figure 8 gives the repeating ring pattern 463815 . Extending this same experiment to $\mathrm{t}=16$, it appears at first glance that the pattern 463815 simply keeps on repeating indefinitely, and suggesting oscillations with period 6 times the original period. However, looking closely at the rings we see that the rings 463815 have some persistent structure, for example, ring 3 in Figure 9 actually consists of 3 rings - the same as in the time window $14<t<16$. On the other hand, looking at ring 3 over the period $6<t<12$ we see a thick solid ring (i.e. consists of many rings) with some simple rings attached to it.

At $D=1.9270 \times 10^{12}$, oscillations appear quite chaotic and seem to persist indefinitely. See Figures 10 and 11 . When $D>1.9275 \times 10^{12}$, oscillations eventually lead to extinction - even if one starts with persistent oscillations at slightly lower $D$.

\section{Conclusions}

Observe the difference in slopes of the curves representing steady solutions in Figures 3 and 4. Note also that the region of stable solutions with a complex leading eigenvalue (thick dashed line) in Figure 4 is not observable in Figure 3 - it reduces to a point. Therefore, the whole period doubling cascade in Figure 4 would look like a vertical line starting at the bifurcation point A in Figure 3. Figure 3 itself shows only a small part of all the relevant steady states. Therefore one could say that this periodic doubling cascade occurs in a very small region.

As one might expect, the period doubling cascade near point B in Figure 3 is almost exactly the same with just one notable difference: $D^{*}<D_{1}$ at point $\mathrm{B}$. This means that small perturbations of the unstable steady solutions result either in SPO or flame 


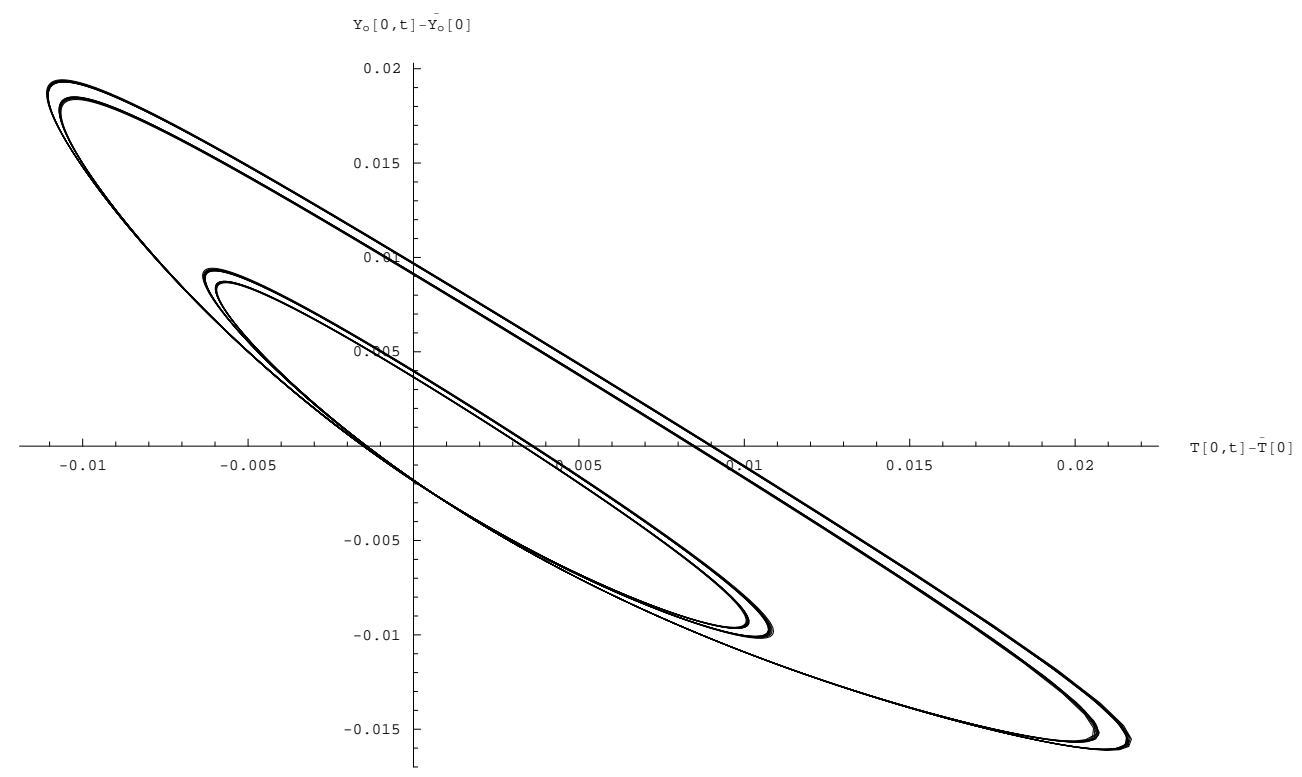

Figure 7: $D=1.9245 \times 10^{12}$ - same experiment as in Figure 6 but with the initial transition period omitted.

extinction - period doubling cannot be observed. This is why period doublings were not noticed in [13]. To obtain the whole cascade one needs to start with initial conditions closer to the invariant periodic manifold.

Point $\mathrm{A}$ is on the tail of the $\mathrm{S}$-curve, and point $\mathrm{B}$, at slightly larger $R$, is on the island [13]. As $R$ increases, the island shrinks and at a certain value of $R$ the bifurcation is no longer supercritical [13]. Therefore the region of the period doubling cascade has to shrink further as $R$ increases, disappearing at the point where we lose supercriticality. As $R$ decreases, the region of unstable states on the tail shrinks and eventually disappears. Period doubling cascades appearing at the end of the SPO look just like the one described above, except near the point where the region of unstable states disappears.

The above results are for $T_{a}=3$. As $T_{a}$ increases, things appear to remain qualitatively the same - the magnitudes change a lot [13].

As $T_{a}$ approaches 1 things change a lot. For example, unstable states appear on the tail of the S-curve like before, however, perturbations of all of them lead to SPO [13] there are no period doublings. When $T_{a}>1$, but $T_{a}$ is close to 1 , the islands form like in the above case. However, the bifurcations are subcritical on the island [13]. Hence there are no period doublings on islands. When $T_{a}=1$ there are no islands [13].

It was shown that the regions of period doubling cascades are very small and perhaps hard to find numerically. However, from the experimental point of view (see [6]), this may not be that serious if one can set up a flame sheet. One reason is that the natural way to make an experiment is the following: start with a flame and keep on increasing $D$ slowly. If the flame does not go out then $R$, the ratio of characteristic chemical and radiation time scales, is too small. If the flame does go out, then just before the extinction one should see the period doubling cascade, provided that $T_{a}$ is large enough (see above). Note that when $T_{a}=3$ then $D_{3}$ is about $4 \%$ larger than $D_{0}$, which seems to be a large 


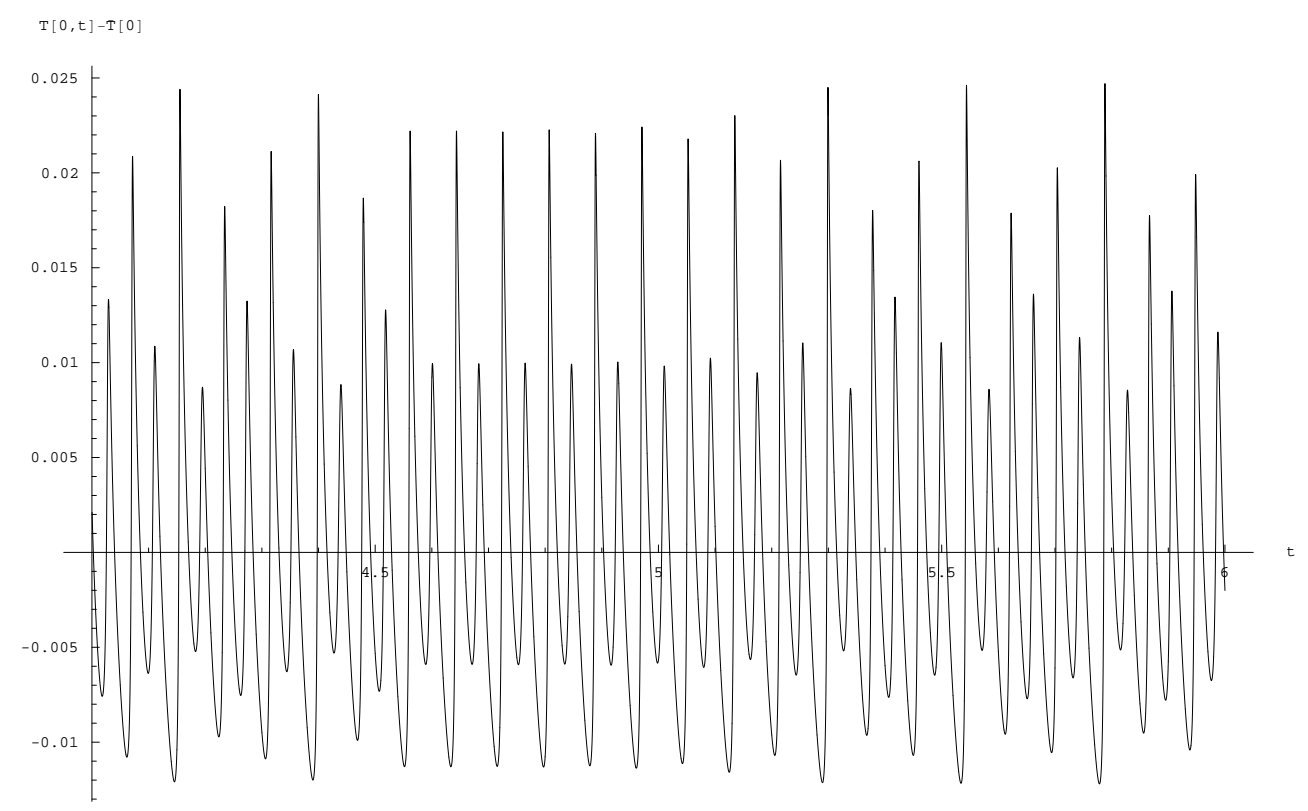

Figure 8: $D=1.9260 \times 10^{12}$

enough difference to be realizable.

\section{References}

[1] Cheatham S and Matalon M, Heat Loss and Lewis Number Effects on the Onset of Oscillations in Diffusion Flames, Proceedings of the 26th Symposium on Combustion, 26 (1996), 1063-1070

[2] Cheatham S and Matalon M, A General Asymptotic Theory of Diffusion Flame with Application to Cellular Instability, J. Fluid Mech., 414(2000), 105-144

[3] Christiansen E W, Tse S D and Law C K, A Computational Study of Oscillatory Extinction of Spherical Diffusion Flames, Combustion and Flame, 134(2003), 327-337

[4] Feigenbaum M J, The universal metric properties of nonlinear transformations, $J$. Stat. Physics, 21(6)(1979), 669-706

[5] Frankel M, Roytburd V and Sivashinsky G, A Sequence of Period Doublings and Chaotic Pulsations in a Free Boundary Problem Modeling Thermal Instabilities, SIAM J. Appl. Math., 54(4)(1994), 1101-1112

[6] Gorman M, El-Hamdi M and Robbins K A, Chaotic Dynamics Near the Extinction Limit of a Premixed Flame on a Porous Plug Burner Combust. Sci. Tech. 98(1994), 47-56

[7] Henrick A K, Aslam T D and Powers J M, Simulation of pulsating onedimensional detonations with true fifth order accuracy, J. Comp Physics 213(1)(2006), 311-329 


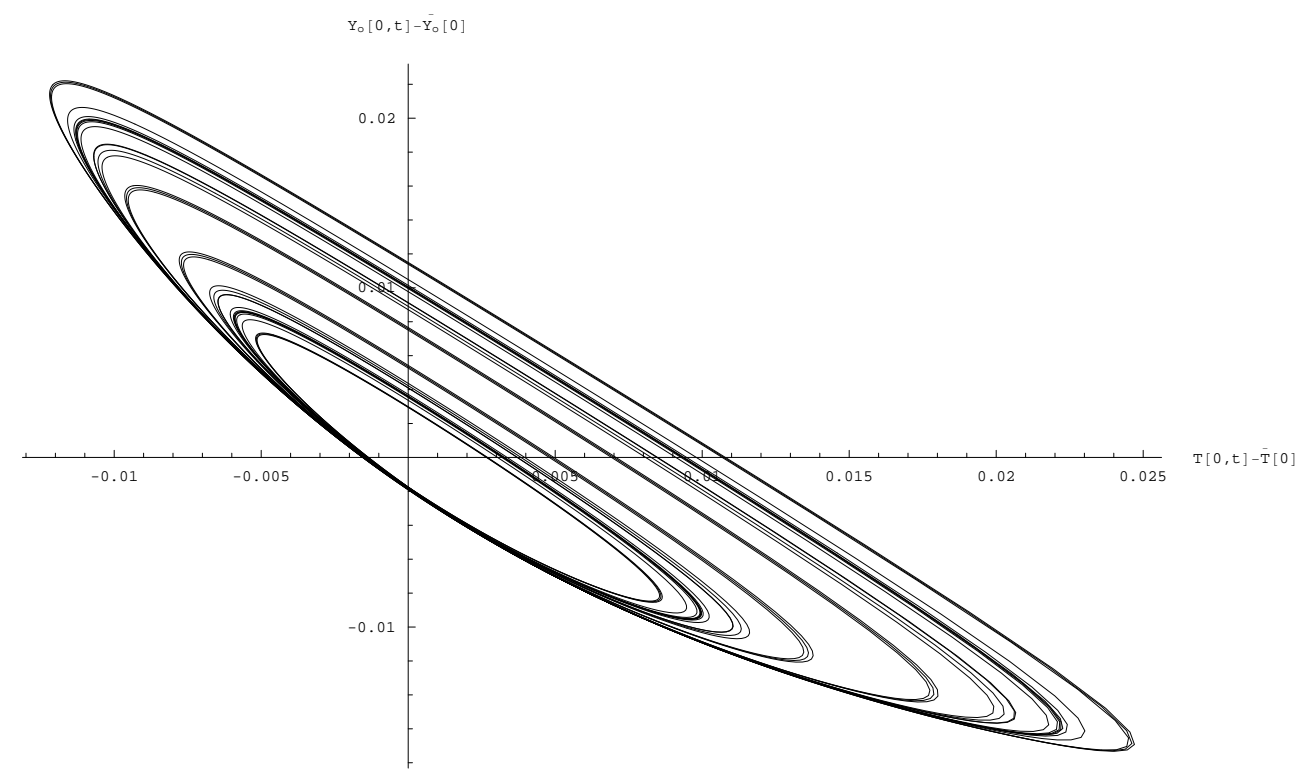

Figure 9: $D=1.9260 \times 10^{12}$ - same experiment as in Figure 8 but with $t>4.5$.

[8] Joulin And Sivashinsky, Combust. Sci. Tech. 55(1987), 83-88

[9] Kim J S, Linear Analysis of Diffusional-Thermal Instability in Diffusion Flames with Lewis Numbers Close to Unity, Comb. Th. Model., 1(1997), 13

[10] Kim J S, LeE S R, Diffusional-Thermal Instability in Strained Diffusion Flames with Unequal Lewis Numbers, Comb. Th. Model., 3(1999), 123

[11] Kim J S, Williams F A, Ronney P D, Diffusional - thermal instability of diffusion flames J. Fluid Mechanics, 327 (1996) 273-301

[12] Margolis S and Matkowsky B, Flame propagation in channels: secondary bifurcation to quasiperiodic pulsations SIAM J. Appl. Math., 45(1985), 93-129

[13] Miklavčič M, Moore A B, Wichman I S, Oscillations and Island Evolution in Radiating Diffusion Flames Combust. Theory Modelling, 9 (2005) 403-416.

[14] Sohn C H, Chung S H, Kim J S, Instability-Induced Extinction of Diffusion Flames Established in the Stagnant Mixing Layer, Combust. Flame, 117(1999) 404-412

[15] Sohn C H, Kim J S, Chung S H, Maruta K, Nonlinear Evolution of Diffusion Flame Oscillations Triggered by Radiative Heat Loss, Combust. Flame, 123(2000) 95-106

[16] T'IEN J S, Diffusion Flame Extinction at Small Stretch Rates: The Mechanism of Radiative Loss, Combustion and Flame, 65(1986), 31-34

[17] Vance R, MiklavČIČ M, Wichman I S, On stability of one dimensional diffusion flames, Combust. Theory Modelling, 5 (2001) 147-161 


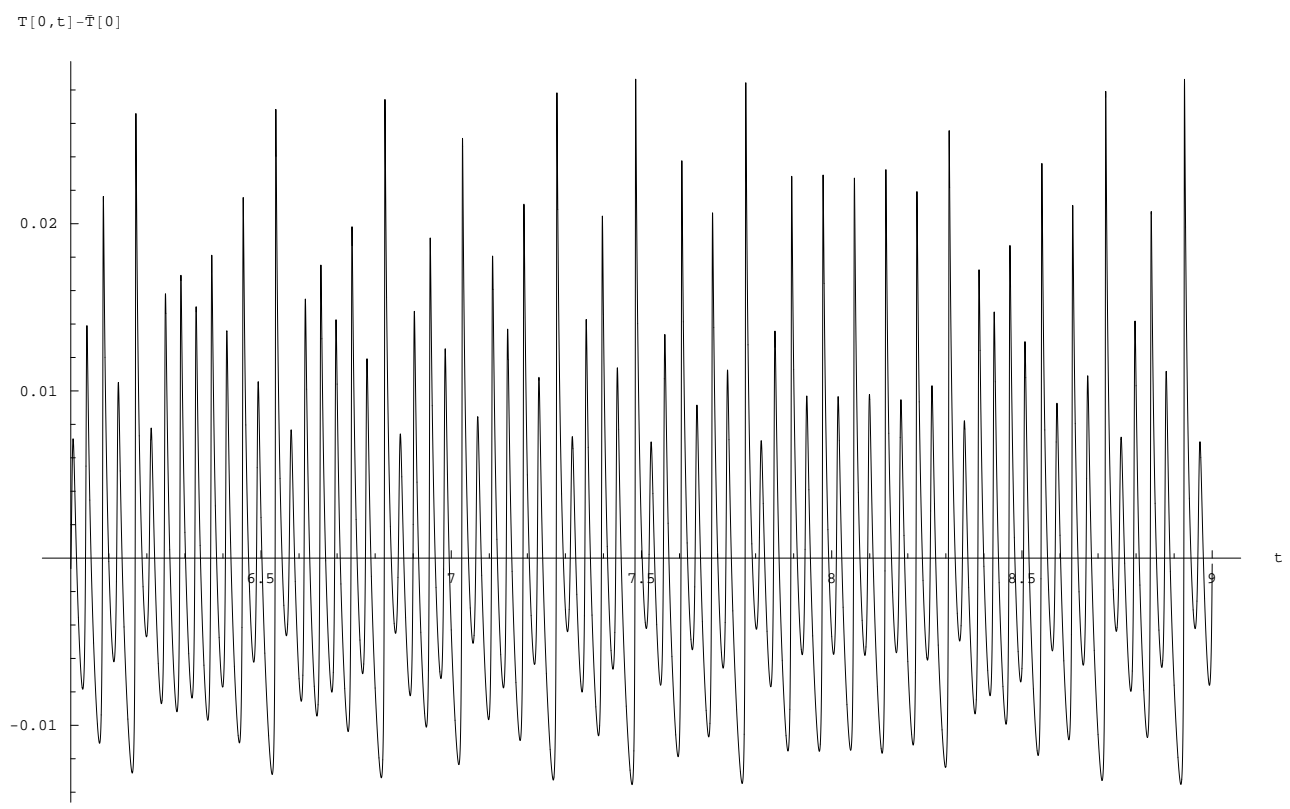

Figure 10: $D=1.9270 \times 10^{12}$

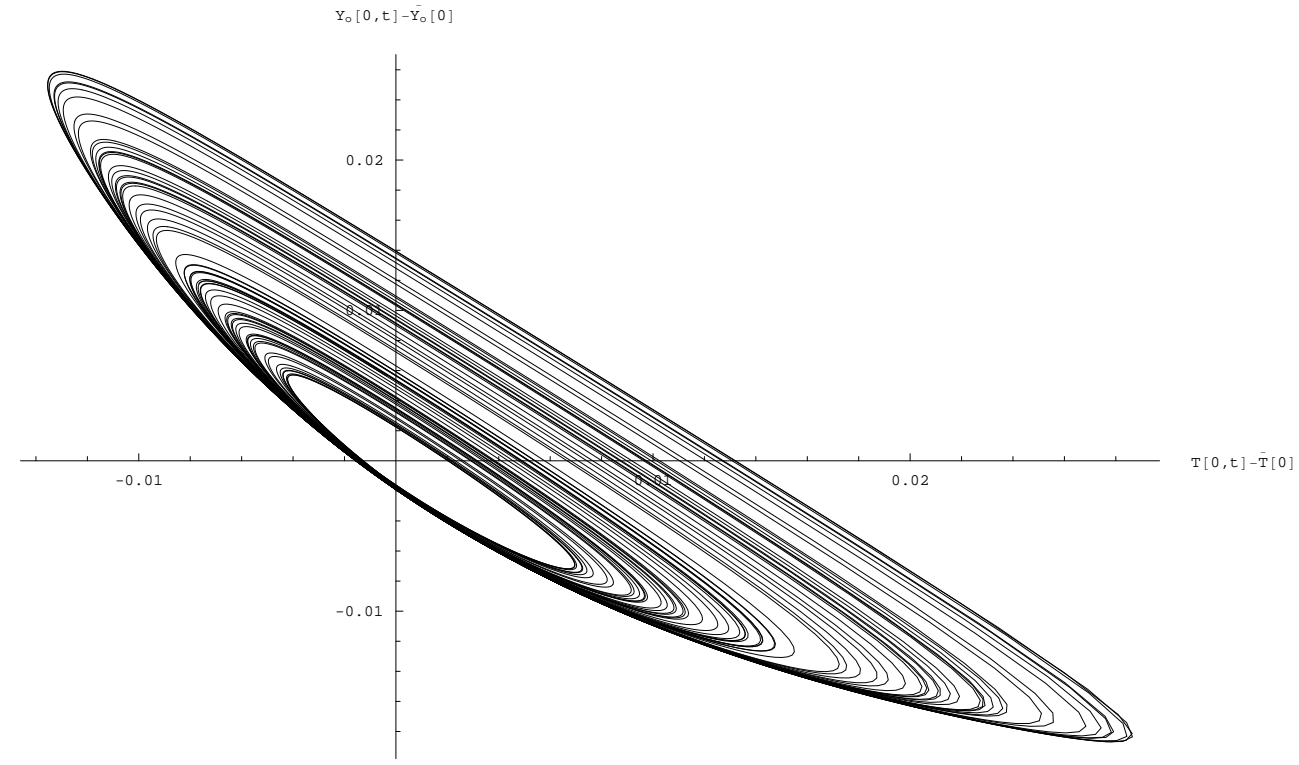

Figure 11: $D=1.9270 \times 10^{12}$ - same experiment as in Figure 10 . 